\title{
Ex vivo efficacy demonstration of a laser fenestration system for endovascular abdominal aortic aneurysm repair (EVAR)
}

Roberta Piazza, Filippo Micheletti, Sara Condino, Giada Magni, Raffaella Nice Berchiolli, et al.

Roberta Piazza, Filippo Micheletti, Sara Condino, Giada Magni, Raffaella Nice Berchiolli, Paolo De Simone, Vincenzo Ferrari, Mauro Ferrari, Francesca Rossi, "Ex vivo efficacy demonstration of a laser fenestration system for endovascular abdominal aortic aneurysm repair (EVAR)," Proc. SPIE 11079, Medical Laser Applications and Laser-Tissue Interactions IX, 1107907 (22 July 2019); doi: 10.1117/12.2527113 


\title{
Ex vivo efficacy demonstration of a laser fenestration system for endovascular aortic aneurysm repair (EVAR)
}

\author{
Roberta Piazza ${ }^{\mathrm{a}, \mathrm{b}}$, Filippo Micheletti*c ${ }^{\mathrm{c}}$, Sara Condino ${ }^{\mathrm{a}, \mathrm{b}}$, Giada Magni ${ }^{\mathrm{c}}$, Raffaella Nice Berchiolli ${ }^{\mathrm{d}}$, \\ Paolo De Simone ${ }^{\mathrm{e}}$, Vincenzo Ferrari ${ }^{\mathrm{a}, \mathrm{b}}$, Mauro Ferrari ${ }^{\mathrm{b}, \mathrm{d}}$, and Francesca Rossi ${ }^{\mathrm{c}}$ \\ ${ }^{a}$ Department of Information Engineering, University of Pisa, Via Caruso 16, Pisa, Italy 56122; \\ ${ }^{\mathrm{b}}$ EndoCAS Center, Department of Translational Research and of New Surgical and Medical \\ Technologies, University of Pisa, Via Paradisa 2, Pisa, Italy 56124; ' Institute of Applied Physics \\ Nello Carrara, National Research Council, Via Madonna del Piano 10, Sesto Fiorentino -FI, Italy \\ 50019; ${ }^{\mathrm{d}}$ Vascular Surgery Unit, Department of Translational Research and of New Surgical and \\ Medical Technologies, Cisanello University Hospital, University of Pisa, Via Paradisa 2, Pisa, Italy \\ 56124; ${ }^{\mathrm{e}}$ Hepatobiliary Surgery and Liver Transplantation, University of Pisa Medical School \\ Hospital, Via Paradisa 2, Pisa, Italy 56124
}

\begin{abstract}
Laser-generated fenestration is an alternative option for the intraoperative and selective modification of a endovascular endograft, especially in cases where patients are unsuitable for a standard endovascular aneurysms repair. Recently, diode laser approach has been proposed as a substitution of mechanical fenestration. In fact, using a near infrared wavelength $(810 \mathrm{~nm})$, the stent graft fabric can be successfully perforated. In this work we report an ex-vivo study providing the harmlessness of laser irradiation effects on biological tissue surrounding the endograft wall. 225 samples of human aortic tissue were irradiated varying energy and pulse duration of an $810 \mathrm{~nm}$ diode laser. Irradiated tissues were analyzed under histological examination. Thermal damage was evidenced in the $7.5 \%$ of the irradiated samples, typically in the contact area between the laser fiber tip and the aortic wall. These experiments suggest that the diode laser can be safely used for the proposed surgical application.
\end{abstract}

Keywords: laser fenestration, diode laser, endovascular aneurysm repair

Short abstract: In situ laser fenestration of endovascular graft enables a safe patient's tailored graft, with minimal side effects and provides a simple and cost-effective way to obtain fenestrated stent grafts.

\section{INTRODUCTION}

The minimally invasive endovascular aneurysm repair (EVAR) is a catheter-based surgical technique consisting of the deployment of an endograft within the aneurismal sac. A bi-dimensional imaging modality based on X-ray exposure (fluoroscopy) is currently used to visualize and guide endovascular instruments within patient's vasculature.

Despite progresses in EVAR, exclusion of aneurysms involving renal, visceral, and/or supra-aortic branches remains a challenge in case of certain anatomical configuration of the aneurysm. Indeed, the proper deployment of a standard endoprosthesis can require prolonged surgical time and enhanced amount of X-ray radiation dose for positioning the stent graft without blocking the passage of blood flow through branched vessels. For this reason, patient specific fenestrated grafting have been developed in order to maintain patency to collateral arteries. However they are technically challenging since the customized holes should be perfectly aligned with the branched vessel origins in order to preserve blood flow. Moreover, customized fenestrated grafts are expensive and require significant pre-planning. These aspects make such devices not immediately available for acute syndromes and for emergency situations.

In situ mechanical perforation of the endograft after its deployment has been proposed ${ }^{1-8}$, in order to perform a tailored surgery on patient's anatomy and to improve the patients eligibility criteria for this surgical approach'.

*f.micheletti@ifac.cnr.it; phone+39055 6445; bnlab.ifac.cnr.it

Medical Laser Applications and Laser-Tissue Interactions IX, edited by Lothar D. Lilge, Carsten M. Philipp, Proc. of SPIE Vol. 11079, $1107907 \cdot$ C 2019 SPIE · CCC code: 1605-7422/19/\$21 · doi: 10.1117/12.2527113 
However, the limit of mechanical fenestration is its reliability in avoiding injuries if the applied force to create the hole, i.e. pushing a needle through the graft fabric, is excessive and thus a damage in aortic tissues can occur. In addition, mechanical fenestration approach is not selective and if the targeting of the fenestration site is not accurate there is a risk of damaging the aortic wall too. For this reason, in recent years, physical methods employing the transferred energy, such as a laser system, have been considered ${ }^{10-13}$. The nature of laser-tissue interaction varies depending on the type of laser energy being absorbed and the material absorbing the energy ${ }^{14}$. We recently proposed an ad hoc build endovascular laser tool emitting in the near infrared wavelength $(810 \mathrm{~nm})$ to fenestrate the graft material without damaging the blood tissue $^{15,16}$, and a computer-assisted method to precisely guide this fenestration tool to the target ${ }^{17,18}$.

According to literature, there are several studies regarding different laser systems to create fenestrations, and most of them have focused on the feasibility of the new intraoperative procedure ${ }^{19}$ and on the evaluation of mechanical integrity of the fenestrated fabric ${ }^{20}$. Whereas the absorption of biological tissues to radiation emitted in the near-infrared range is minimized but not zero, there is no work regarding the effects of diode laser on biological tissue that demonstrates the safeness of this new technique for blood vessels.

In this work we present the results of an ex vivo laser fenestration tests on samples of human aortic tissue in order to demonstrate that the diode laser operative conditions, which ensure the fabric fenestration, are safe for the patient's vasculature surrounding the endograft. Our hypothesis is that the diode laser is harmless even in the worst case of direct irradiation of the aorta wall due to an incorrect targeting of the fenestration site.

\section{MATERIALS AND METHODS}

\subsection{Sample collection and preparation}

Anonymized human aortic samples were collected from 7 consenting patients with aortic abdominal aneurysm and 9 cadaveric donors. The study was approved by the Cisanello University Hospital (Azienda Ospedaliero-Universitaria Pisana, A.O.U.P., Pisa, Italy) ethical committee.

The samples were saline solution rinsed to remove excess blood residue. All collected samples were preserved at $+4^{\circ} \mathrm{C}$ until laser irradiation test, conducted within 48 hours of sampling. Circular segments $4 \mathrm{~mm}$ in diameter were cut from each sample using a biopsy punch. One segment from each sample was preserved and used as a control for histological analysis. A multi-well plate was used to collect segments from each sample (Figure 1).
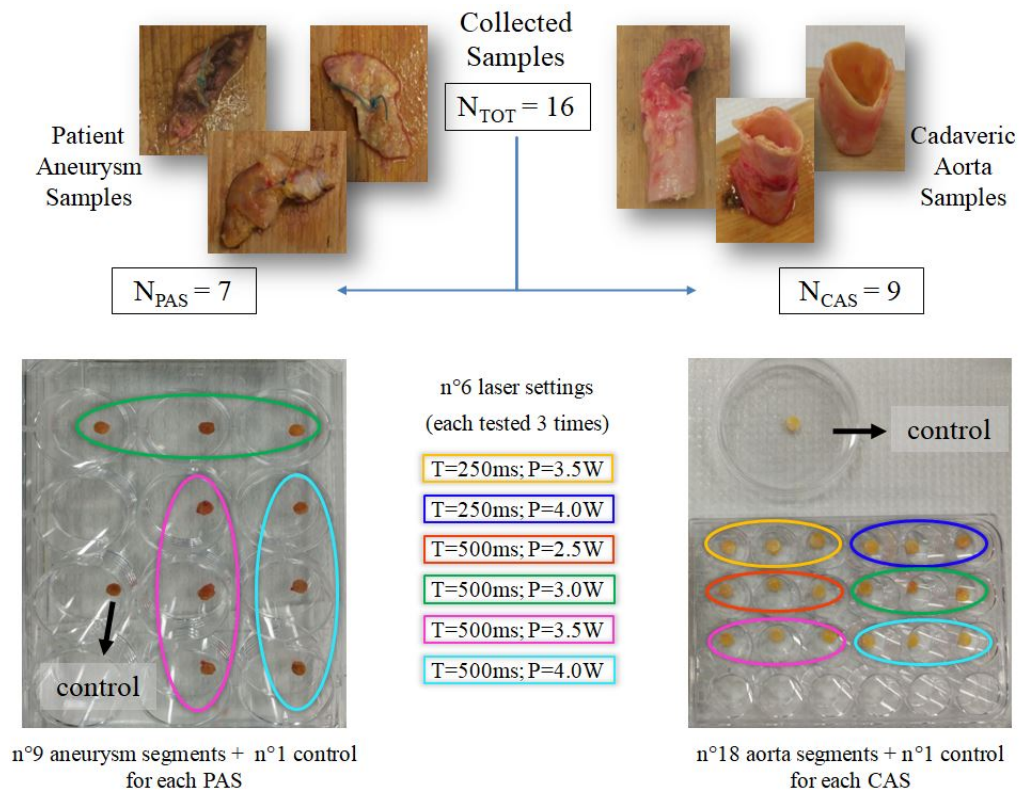

Figure 1. Collection and preparation of aneurysm and aorta samples: the laser conditions were tested 3 times on 3 different segments from the same sample. Due to smaller size, on patient aneurysm samples (on the left), only the three worst irradiation conditions were tested. 


\subsection{Laser irradiation test}

Irradiation tests were performed with 0.22 Numerical Aperture (NA), high power multimode fiber from Thorlabs, Inc. (Newton, New Jersey, USA) $200 \mu \mathrm{m}$ in diameter, connected to a diode laser system (SMARTY A800, DEKA, Italy) with a $810 \mathrm{~nm}$ wavelength and a maximum power of $10 \mathrm{~W}$. The laser fiber was secured to the holder of a 3 axis micropositioner to precisely move the laser tip in perpendicular contact with the surface of the segment. Each segment was completely covered with $2 \mathrm{ml}$ of human whole blood obtained by venipuncture from a hematologically healthy adult (Figure 2).

The following irradiation conditions, which ensure the perforation of the endograft fabric ${ }^{15,16}$, were tested:

- $\quad 250$ ms irradiation time, power 3.5 and $4 \mathrm{~W}$;

- $500 \mathrm{~ms}$ irradiation time, power ranging from 2.5 to $4 \mathrm{~W}$ at a step interval of $0.5 \mathrm{~W}$.

Each irradiation condition was used 3 times on the same sample: i.e. an irradiation condition was tested on 3 segments from the same sample. After laser irradiation, segments were rinsed with physiologic solution for subsequent analysis.
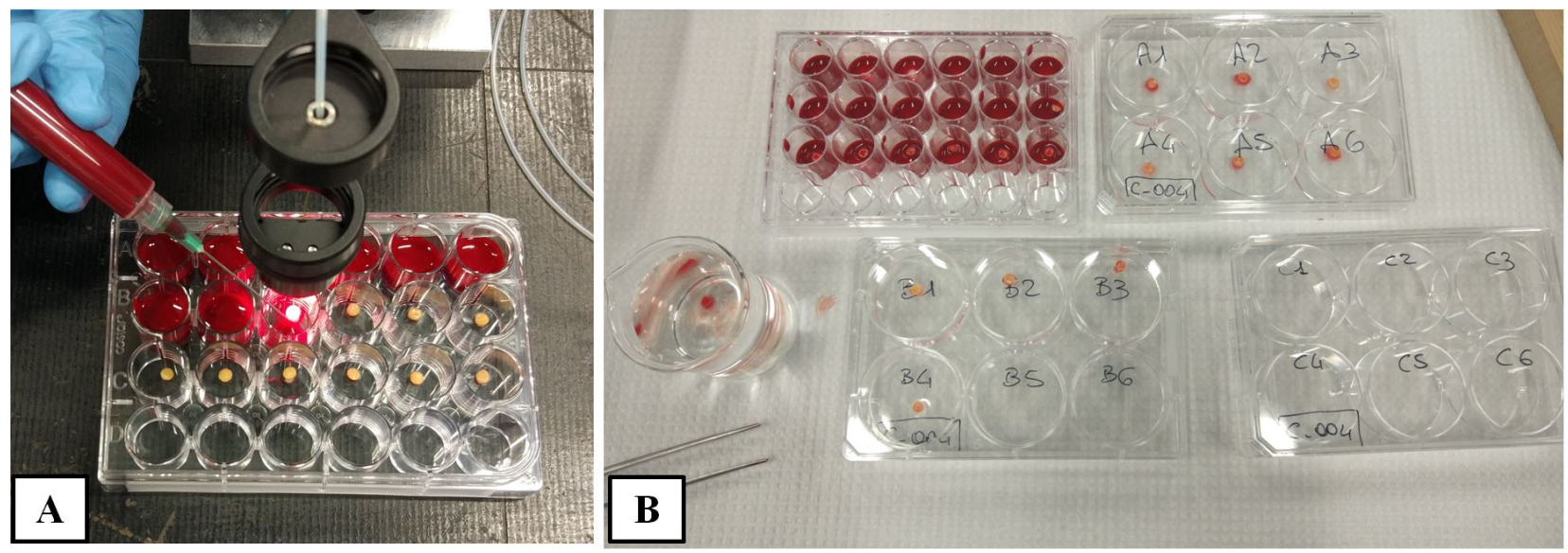

Figure 2. Laser irradiation test: experimental set up (A) and segments preparation (B) for further analysis under microscope.

\subsection{Irradiated samples analysis}

All irradiated segments were evaluated under a stereomicroscope (Nikon SMZ1500, Japan) at a magnification of 10x. H\&E staining technique was used for the analysis under optical microscope (Leica DM500, Leica Microsystems, Buccinasco, Italy), to evidence and observe the entity of any eventual thermal damage. This was determined by comparing the morphological structure of the irradiated segment to the corresponding untreated segment (control) from the same tissue sample.

\section{RESULTS}

Out of 225 irradiated human aortic segments, 17 showed a thermal damage identified by microscope analysis. No damages were observed in trials with an irradiation time of $250 \mathrm{~ms}$ neither in those with a laser power of $2.5 \mathrm{~W}$, indicating that these laser operative parameters can be considered safe for the biological tissue. For all the other irradiation conditions at least one segment from the same sample was damaged. However, most of the segments were not injured by laser irradiation and no irradiation condition has caused tissue damage all three times it was tested.

The histological analysis showed that the damages do not extend beyond the contact point between the fiber tip and the segment surface, thus the tested laser irradiations are harmless for the biological tissue. 

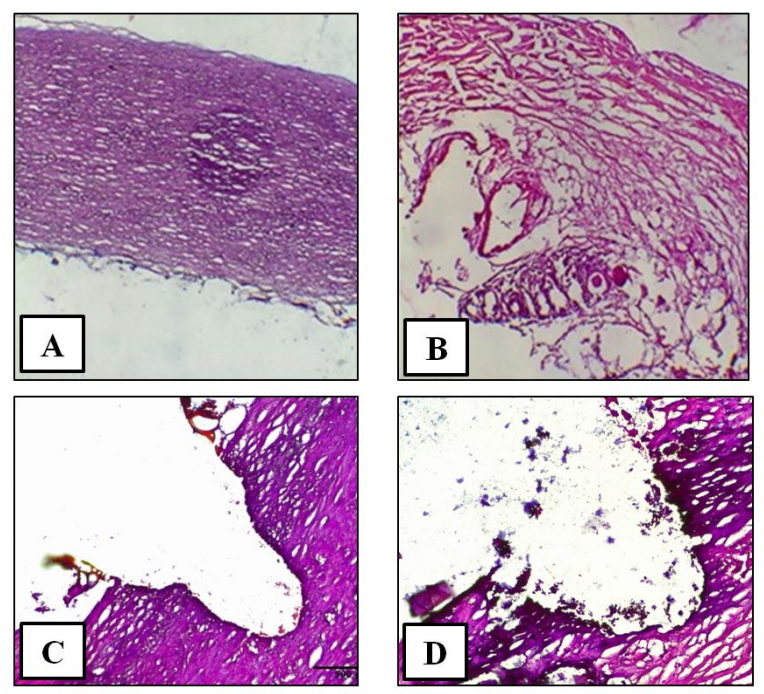

Figure 3. Histological images of: undamaged (A and B) and damaged ( C and D) samples of aorta and aneurysm tissues, respectively.

\section{CONCLUSIONS}

The in situ laser generated fenestration of a standard stent graft is an alternative option to avoid custom fabrication of endoprosthesis and represent an immediate, simple and cost-effective way to obtain fenestrated stent grafts, preserving blood flow to essential visceral arteries, for the treatment of patients unsuitable for a standard EVAR.

This study provides an evaluation of the diode laser technology for the innovative procedure, demonstrating that the same operative irradiation conditions which ensure the fabric fenestration are safe on aortic tissue surrounding the endoprosthesis. Obtained results show that a direct exposure to laser radiation, which represents the worst scenario of accidental contact between laser tip and aortic wall, can generate damage which does not extend beyond the contact point, thus the diode laser irradiation is harmless for the biological tissue.

Currently, the reliability of the in situ fenestration technique is principally limited by difficulties in targeting the proper fenestration site under fluoroscopic control.

Future works will be focused on performing the in situ diode laser fenestration procedure with a 3D navigation system ${ }^{21}$,

${ }^{22}$ to precisely guide the fenestration tool to the target site. Deformable and rigid phantoms $\mathrm{s}^{23-25}$ will be used for the testing session. Moreover, intraoperative data ${ }^{26-28}$ and predictive arterial deformation models ${ }^{29-31}$ could be considered for updating the virtual model of patient's anatomy, which is currently static, in order to compensate the heartbeat and breathing movements.

\section{ACKNOWLEDGEMENTS}

The research leading to these results has been partially supported by the project "Electromagnetic guided in situ laser fenestration of endovascular endoprosthesis" (RF-2011-02350455) funded by the Italian Ministry of Health and Regione Toscana through the call "Ricerca Finalizzata 2011-2012" and by the project "VIVIR: Virtual and Augmented Reality Support for Transcatheter Valve Implantation by using Cardiovascular MRI" (PE-2013-02357974) funded by the Italian Ministry of Health through the call "Ricerca Finalizzata 2013". 


\section{REFERENCES}

[1] J. Bismuth, C. Duran, and H. T. Hassoun, "In situ fenestration for branch vessel preservation during EVAR," Methodist Debakey Cardiovase J, 8(4), 33-36 (2012).

[2] T. Kolbel, S. W. Carpenter, H. Diener et al., "Antegrade in situ stent-graft fenestration for the renal artery following inadvertent coverage during EVAR," J Endovasc Ther, 20(3), 289-294 (2013).

[3] R. G. McWilliams, S. J. Fearn, P. L. Harris et al., "Retrograde fenestration of endoluminal grafts from target vessels: feasibility, technique, and potential usage," J Endovasc Ther, 10(5), 946-952 (2003).

[4] R. G. McWilliams, M. Murphy, D. Hartley et al., "In situ stent-graft fenestration to preserve the left subclavian artery," J Endovasc Ther, 11(2), 170-174 (2004).

[5] C. V. Riga, C. D. Bicknell, D. Wallace et al., "Robot-assisted antegrade in-situ fenestrated stent grafting," Cardiovasc Intervent Radiol, 32(3), 522-524 (2009).

[6] B. W. Starnes, "Physician-modified endovascular grafts for the treatment of elective, symptomatic, or ruptured juxtarenal aortic aneurysms," J Vasc Surg, 56(3), 601-607 (2012).

[7] L. W. H. Tse, B. T. Bui, S. Lerouge et al., "In vivo antegrade fenestration of abdominal aortic Stent-Grafts," J Endovasc Ther, 14(2), 158-167 (2007).

[8] G. H. Wheatley, 3rd, "In situ fenestration of the internal iliac artery as a bailout technique associated with endovascular repair of an abdominal aortic aneurysm: long-term follow-up," J Endovasc Ther, 19(6), 716-720 (2012).

[9] H. L. Graves, and B. M. Jackson, "The Current State of Fenestrated and Branched Devices for Abdominal Aortic Aneurysm Repair," Semin Intervent Radiol, 32(3), 304-310 (2015).

[10] S. S. Ahanchi, B. Almaroof, C. L. Stout et al., "In Situ Laser Fenestration for Revascularization of the Left Subclavian Artery During Emergent Thoracic Endovascular Aortic Repair," J Endovasc Ther, 19(2), 226-230 (2012).

[11] E. H. Murphy, J. M. Dimaio, W. Dean et al., "Endovascular Repair of Acute Traumatic Thoracic Aortic Transection With Laser-Assisted In-Situ Fenestration of a Stent-Graft Covering the Left Subclavian Artery," J Endovasc Ther, 16(4), 457-463 (2009).

[12] E. H. Murphy, G. A. Stanley, M. Ilves et al., "Thoracic Endovascular Repair (TEVAR) in the Management of Aortic Arch Pathology," Ann Vasc Surg, 26(1), 55-66 (2012).

[13] R. E. Redlinger, S. S. Ahanchi, and J. M. Panneton, "In Situ Laser Fenestration During Thoracic Endovascular Aortic Repair Is an Effective Method for Left Subclavian Artery Revascularization," J Vasc Surg, 56(6), 1828-1828 (2012).

[14] J. F. Chen, K. R. Kollmeyer, and S. S. Ahn, [Chapter 11 - Laser Atherectomy] W.B. Saunders, Philadelphia (2011).

[15] S. Condino, R. Piazza, F. Micheletti et al., "Electromagnetic Guided In-Situ Laser Fenestration of Endovascular Stent-Graft: Endovascular Tools Sensorization Strategy and Preliminary Laser Testing," MIAR 2016 LNCS, 9805, 72-83 (2016).

[16] F. Micheletti, R. Pini, R. Piazza et al., "In-Situ laser fenestration of endovascular stent-graft in abdominal aortic aneurysm repair (EVAR)," Proc. SPIE 10042, 100420D (2017).

[17] R. Piazza, S. Condino, A. Alberti et al., "Design of a sensorized guiding catheter for in situ laser fenestration of endovascular stent," Comput Assist Surg, 22(1), 27-38 (2017).

[18] R. Piazza, S. Condino, A. Alberti et al., "Using of 3D Virtual Reality Electromagnetic Navigation for Challenging Cannulation in FEVAR procedure," AVR 2017 LNCS, 10325, 221-229 (2017).

[19] J. Qin, Z. Zhao, R. Wang et al., "In Situ Laser Fenestration Is a Feasible Method for Revascularization of Aortic Arch During Thoracic Endovascular Aortic Repair," J Am Heart Assoc, 6(4), (2017).

[20] J. Lin, N. Parikh, N. Udgiri et al., "Laser Fenestration of Aortic Stent-Grafts Followed by Noncompliant vs Cutting Balloon Dilation: A Scanning Electron Microscopy Study," J Endovasc Ther, 25(3), 397-407 (2018).

[21] S. Condino, E. M. Calabro, A. Alberti et al., "Simultaneous tracking of catheters and guidewires: comparison to standard fluoroscopic guidance for arterial cannulation," Eur J Vasc Endovasc Surg, 47(1), 53-60 (2014).

[22] S. Condino, V. Ferrari, C. Freschi et al., "Electromagnetic navigation platform for endovascular surgery: how to develop sensorized catheters and guidewires," Int J Med Robot, 8(3), 300-310 (2012).

[23] S. Condino, M. Carbone, V. Ferrari et al., "How to build patient-specific synthetic abdominal anatomies. An innovative approach from physical toward hybrid surgical simulators," Int J Med Robotics Comput Assist Surg, 7(2), 202-213 (2011). 
[24] S. Condino, K. Harada, N. N. Pak et al., "Stomach simulator for analysis and validation of surgical endoluminal robots," Appl Bionics Biomech, 8(2), 267-277 (2011).

[25] S. Sinceri, M. Carbone, M. Marconi et al., "Basic Endovascular Skills Trainer: A surgical simulator for the training of novice practitioners of endovascular procedures," Proc IEEE Eng Med Biol Soc, 5102-5105 (2015).

[26] G. Turini, S. Condino, M. Postorino et al., "Improving Endovascular Intraoperative Navigation with RealTime Skeleton-Based Deformation of Virtual Vascular Structures," AVR 2016 LNCS, 9769, 82-91 (2016).

[27] S. Parrini, L. Zhang, S. Condino et al., "Automatic carotid centerline extraction from three-dimensional ultrasound Doppler images," Proc IEEE Eng Med Biol Soc, 5089-5092 (2014).

[28] L. Zhang, S. Parrini, C. Freschi et al., "3D ultrasound centerline tracking of abdominal vessels for endovascular navigation," Int J Comput Assist Radiol Surg, 9(1), 127-135 (2014).

[29] D. Perrin, P. Badel, L. Orgéas et al., "Patient-specific numerical simulation of stent-graft deployment: Validation on three clinical cases," J Biomech, 48(10), 1868-1875 (2015).

[30] J. Gindre, A. Bel-Brunon, M. Rochette et al., "Patient-Specific Finite-Element Simulation of the Insertion of Guidewire During an EVAR Procedure: Guidewire Position Prediction Validation on 28 Cases," IEEE Trans Biomed Eng, 64(5), 1057-1066 (2017).

[31] M. Conti, M. Marconi, G. Campanile et al., "Patient-specific finite element analysis of popliteal stenting," Meccanica, 52(3), 633-644 (2017). 